\title{
Abdominal Apoplexy after Pulmonary Lobectomy for Lung Cancer
}

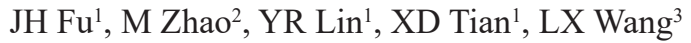

\begin{abstract}
Abdominal apoplexy is a rare clinical entity, and its clinical manifestations are diverse. This case report is of a 52-year-old man who developed right upper abdominal pain with unstable haemodynamics 32 hours after right upper pulmonary lobectomy for lung carcinoma. Abdominal computed tomography showed a ruptured right gastric artery aneurysm.
\end{abstract}

Keywords: abdominal apoplexy, bleeding, gastric artery aneurysm, lung cancer, pulmonary lobectomy

\section{Apoplejía abdominal tras una lobectomía pulmonar por cáncer de pulmón}

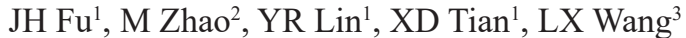

\begin{abstract}
RESUMEN
La apoplejía abdominal es una entidad clínica rara, y sus manifestaciones clínicas son diversas. Este es un reporte de caso de un hombre de 52 años que presentó dolor abdominal superior derecho con hemodinámica inestable, 32 horas después de una lobectomía pulmonar superior derecha por carcinoma del pulmón. La tomografía computarizada abdominal mostró una ruptura de aneurisma de la arteria gástrica derecha.
\end{abstract}

Palabras clave: Apoplejía abdominal, sangramiento, aneurisma de la arteria gástrica, cáncer de pulmón, lobectomía pulmonar

West Indian Med J 2018; 67 (3): 289

\section{INTRODUCTION}

Abdominal apoplexy is clinically rare and is characterized by spontaneous vascular spasm, embolism, and rupture of abdominal viscera, resulting in acute abdominal pain. Abdominal apoplexy was first reported in 1909 and was later termed as stomach stroke in $1931(1,2)$. So far, fewer than 100 cases have been reported around the world (1-5). We report herein the first case of abdominal apoplexy after surgical treatment of lung cancer.

From: 'Department of Cardiothoracic Surgery and Cardiology, Liaocheng People's Hospital and Liaocheng Clinical School of Taishan Medical University, Liaocheng, Shandong Province, People's Republic of China 252000, ${ }^{2}$ Department of Scientific Research and Educating Section, Liaocheng People's Hospital and Liaocheng Clinical School of Taishan Medical University, Liaocheng, Shandong Province, People's Republic of China 252000 and ${ }^{3}$ School

\section{CASE REPORT}

This retrospective report was approved by the institutional review board of Liaocheng People's Hospital, Liaocheng, Shandong, People's Republic of China. Written consent was obtained from the patient for this report. The patient was a 52-year-old man who presented with a one-month history of productive cough without haemoptysis. He had no previous cardiovascular or respiratory history. Chest computed tomography

of Biomedical Sciences, Charles Sturt University, Wagga Wagga, NSW 2678, Australia.

Correspondence: Professor L Wang, Department of Cardiothoracic Surgery and Cardiology, Liaocheng People's Hospital, Liaocheng, Shandong Province, People's Republic of China 252000. Email: lwang@csu.edu.au 
(CT) showed a $3.3 \times 2.6 \mathrm{~cm}$ soft tissue mass in the right upper lung lobe with a CT value of 57-69 HU (Fig. 1A, B). Bronchoscopy revealed a neoplasm in the anterior segment of the right upper lobe, partially blocking the bronchial lumen. A lung brush specimen showed squamous cell carcinoma.

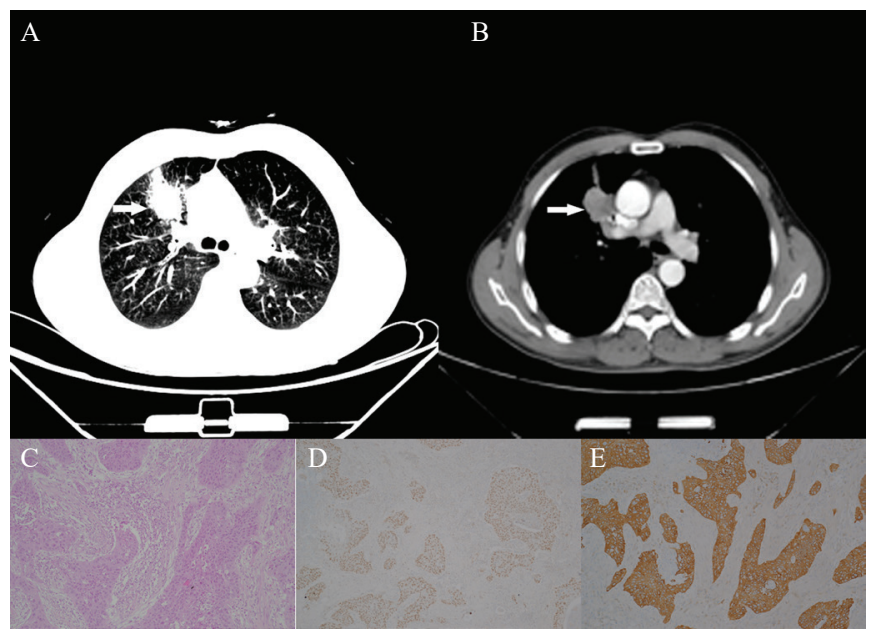

Fig. 1: Chest computed tomography before lobectomy (A, B) and tumour staining after lobectomy $(\mathrm{C}, \mathrm{D}, \mathrm{E})$. A: lung window of chest computed tomography. B: mediastinal window of chest computed tomography. C: haematoxylin-eosin staining of lung squamous cell carcinoma $(\times 10)$. D: P63 staining (nuclear staining) $(\times 10)$. E: CK5/6 staining (cytoplasmic staining) $(\times 10)$.

Exploratory thoracotomy revealed a tumour in the anterior segment of the right upper lung lobe. Lymph node excision plus resection of the right upper lung lobe was carried out uneventfully. Postoperatively, the pathological diagnosis was squamous cell carcinoma with no evidence of metastasis in the lymph nodes (Fig. 1C, D).

The patient reported intermittent pain to the right of the umbilicus 32 hours after the operation. The abdominal pain worsened about 30 minutes after the onset, forcing him into a right lateral position. He then became confused and clammy, and was cold and pale in the face and limbs. His heart rate increased to 120 beats/minute, and his blood pressure was not measurable (too low).

The chest tube inserted following the lobectomy was patent and drained a small amount of pale red liquid, and his upper abdomen was slightly distended. His entire abdomen was tender, but no rebound tenderness was observed. He was treated with dopamine infusion to support the blood pressure. His confusion was improved, but severe abdominal pain remained along with nausea and vomiting. His heart rate and blood pressure were 161 beats/minute and 70/40 $\mathrm{mmHg}$, respectively. He was diagnosed with postoperative shock and abdominal pain of unknown origin. The patient was transferred to the intensive care unit, where blood analysis showed the following: white blood cell count, $31.47 \times 10^{9} / \mathrm{L}$; red blood cell count, $3.27 \times 10^{12} / \mathrm{L}$; and haemoglobin, $108 \mathrm{~g} / \mathrm{L}$. Abdominal ultrasound showed a low to echo-free zone on the right and upper side of the umbilicus with ascites. Abdominal CT showed an uneven distal right gastric artery lumen, suggesting right gastric artery rupture with abdominal and pelvic haematomas (Fig. 2A, B).

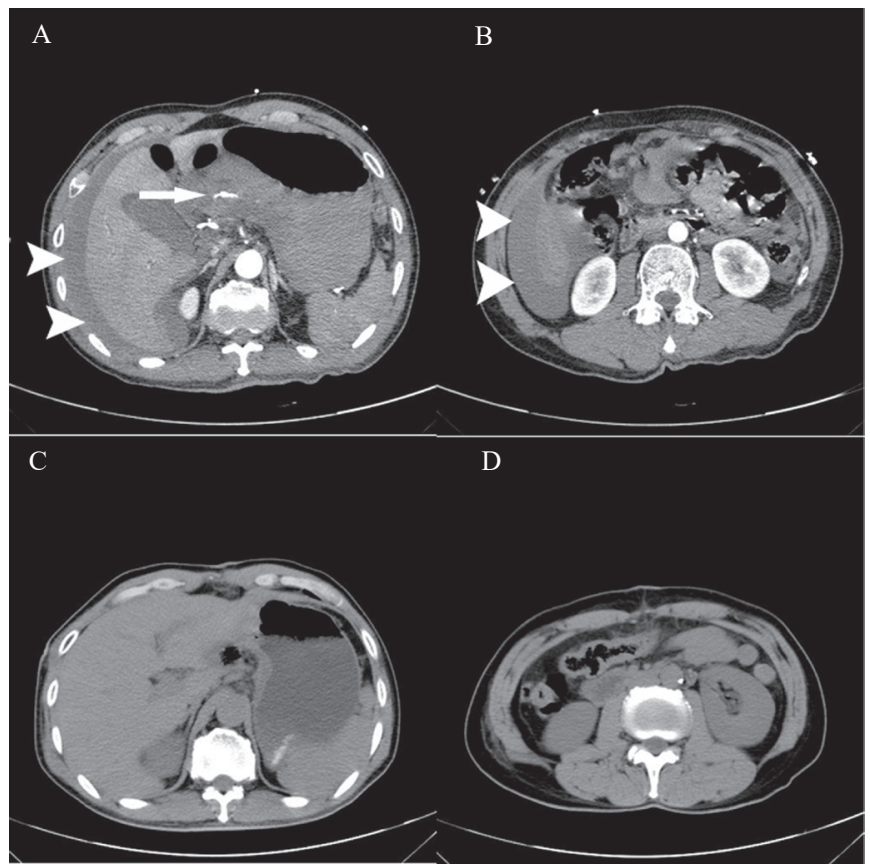

Fig. 2: Abdominal computed tomography scan before laparotomy (A, B) and seven days after laparotomy (C, D). A: ruptured blood vessels in lesser curvature of stomach (arrow). B: blood in abdominal and pelvic cavities (arrowheads). C, D: disappearance of haematomas in the abdominal and pelvic cavities.

Exploratory laparotomy was performed because of the abdominal bleeding and continuously decreasing haemoglobin level. Approximately $3000 \mathrm{ml}$ of blood was removed from the abdominal and pelvic cavities. The omental bursa contained a large amount of blood clots, and the vessels of the lesser curvature were bleeding. After the haemorrhage and blood clots were cleared, the lesser sac was opened, the left gastric artery was ligated, and the vessels of the lesser curvature were sutured in an interrupted pattern. A shunt catheter was placed under the liver to drain blood and fluid. One week later, abdominal CT showed no effusion in the abdominal or pelvic cavity (Fig. 2C, D). The patient recovered uneventfully without abdominal discomfort. He was discharged from the hospital two weeks postoperatively. 


\section{DISCUSSION}

The bleeding site in patients with abdominal apoplexy is most commonly found at the second- or third-level branch of the abdominal aorta, such as the left or right gastric artery. The causes of abdominal apoplexy are not entirely clear, but the condition may be related to the following factors. Firstly, hypertension and atherosclerosis, which are more common in elderly patients, may damage the normal structure of the blood vessel wall, which may cause dissection aneurysm and rupture upon a sudden increase in blood pressure or abdominal pressure (3).

Secondly, arterial abnormalities or congenital defects coupled with a sudden change in blood flow resistance can result in atraumatic spontaneous rupture of the arterial aneurysm. This usually occurs in young patients (3). In addition, abdominal apoplexy may also be induced by systemic infection (eg subacute bacterial endocarditis). The predisposing factors for abdominal apoplexy include sneezing, coughing and even bending sideways, all of which can cause mild to severe abdominal muscle contraction. This results in a sudden increase in omental pressure and resistance to blood flow, causing vascular rupture and bleeding.

Abdominal apoplexy has no specific clinical manifestations. The clinical onset is usually abrupt. It is more common in men aged $>50$ years; such patients often present with acute abdominal pain and bleeding symptoms $(3,4)$. Ultrasound is the first choice of imaging studies. Computed tomography also has high diagnostic value, but patients need to be haemodynamically stable to have this test. Selective abdominal aorta radiography is a better means of diagnosis and treatment because it can directly reveal ruptured blood vessels and provides interventional therapy. However, it is appropriate only in stable patients with minimal abdominal bleeding. If disease progression is rapid or if the diagnosis is unclear, surgical exploration should be performed in a timely manner (4).
Early surgical exploration should be performed upon identification of the abdominal bleeding $(4,5)$. Early exploratory laparotomy and haemostasis offer the best prognosis. Intraoperative identification of all haemorrhaging vessels and precise suturing or excision of the lesions should be performed; parts of the clot or haematoma are often the source of bleeding. If the haemorrhaging vessels cannot be located, a drainage tube should be placed, and the patient should be dynamically observed for internal bleeding.

Our patient developed symptoms a day after pulmonary lobectomy. During the operation, we needed to collapse each lobe of the right lung, and postoperative lung recruitment was also required. Such patients often produce more phlegm (sometimes forced), resulting in a sudden increase in abdominal pressure and vascular resistance. These may be factors related to the development of intra-abdominal bleeding. Abdominal CT showed a bleeding right gastric aneurysm, suggesting that this was the cause of the abdominal stroke. Based on the positive response to anti-shock treatment, decisive exploratory laparotomy with haemostasis was the key factor in the success of the treatment.

\section{AUTHORS' NOTE}

The authors have no conflicts of interest to declare.

\section{REFERENCES}

1. Watkins GL. Abdominal apoplexy. Ann Surg 1962; 155: 153-60

2. Kleinsasser LJ. Abdominal apoplexy. Report of two cases and review of the literature. Am J Surg 1970; 120: 623-8.

3. Dvorak A, Gazzaniga A. Dissecting aneurysm of the gastroduodenal artery: anatomic basis for the clinical syndrome of abdominal apoplexy. Ann Surg 1969; 169: 425-8.

4. Carr SR, Dinsmore RC, Wilkinson NW. Idiopathic spontaneous intraperitoneal hemorrhage: a clinical update on abdominal apoplexy in the year 2001. Am Surg 2001; 67: 374-6.

5. Jadav M, Ducheine Y, Brief D, Carter L, McWhite T, Hardy J. Abdominal apoplexy: a case study of the spontaneous rupture of the gastroepiploic artery. Curr Surg 2004; 61: 370-2. 